\title{
Low-Drift Closed-Loop Fiber Optic Gyroscope of High Scale Factor Stability Driven by Laser With External Phase Modulation
}

\author{
Jingtao YAN, Lijun MIAO*, Heliang SHEN, Xiaowu SHU, \\ Tengchao HUANG, and Shuangliang CHE
}

State Key Laboratory of Modern Optical Instrumentation, Zhejiang University, Hangzhou 310027, China

${ }^{*}$ Corresponding author: Lijun MIAO_Ｅ-mail: mlj000000@zju.edu.cn

\begin{abstract}
In view of the poor scale factor stability of the interferometric fiber optic gyroscope (IFOG), it is a creative method to use laser to drive the IFOG for its better frequency stabilization characteristics instead of the broadband light source. As the linewidth of laser is narrow, the errors of coherent backscattering, polarization coupling, and Kerr effect are reintroduced which cause more noise and drift. This paper studies laser spectrum broadening based on external phase modulation of Gaussian white noise (GWN). The theoretical analysis and test results indicate that this method has a good effect on spectrum broadening and can be used to improve the performance of the laser-driven IFOG. In the established closed-loop IFOG, a four-state modulation (FSM) is adopted to avoid temperature instability of the multifunction integrated-optic chip (MIOC) and drift caused by the electronic circuit in demodulation. The experimental results show that the IFOG driven by broadened laser has the angular random walk noise of $0.0038 \% / \mathrm{h}$ and the drift of $0.017 \% / \mathrm{h}$, which are $62 \%$ and $66 \%$ better than those without modulation respectively, of which the drift has reached the level of the broadband light source. Although the noise still needs further reduction, its scale factor stability is $0.38 \mathrm{ppm}$, which has an overwhelming advantage compared with the traditional IFOG.
\end{abstract}

Keywords: Fiber optic gyroscope; laser linewidth; Gaussian white noise; external phase modulation; closed-loop detection

Citation: Jingtao YAN, Lijun MIAO, Heliang SHEN, Xiaowu SHU, Tengchao HUANG, and Shuangliang CHE, "Low-Drift Closed-Loop Fiber Optic Gyroscope of High Scale Factor Stability Driven by Laser With External Phase Modulation," Photonic Sensors, 2022, 12(3): 220304.

\section{Introduction}

As a kind of rotation rate sensor based on the Sagnac effect [1], interferometric fiber optic gyroscope (IFOG) has been developed for over forty years. It is an inertial navigation instrument that is widely used in national defense industries and civilian applications such as aviation, aerospace, and land navigation [2-5]. Compared with its main competitor, ring laser gyroscope (RLG), the IFOG does not need high-precision processing of the optical mirror and strict sealing of the cavity or ignition voltage of several thousand volts. Furthermore, it effectively overcomes the self-locking phenomenon and has the characteristics of simple structure, low cost, small volume, and light weight [6-8]. From the perspective of market, the IFOG has a tendency to replace the RLG

Received: 13 May 2021 / Revised: 26 October 2021

(C) The Author(s) 2022. This article is published with open access at Springerlink.com

DOI: $10.1007 / \mathrm{s} 13320-022-0648-7$

Article type: Regular 
completely. However, the most serious problem needing to solve in this process is to improve the scale factor stability of the IFOG, which is usually driven by the broadband superluminescent diode (SLD) or superfluorescent fiber source (SFS) and its average wavelength stability is much worse than that of laser [9].

In recent years, research groups represented by Stanford University have been devoted to using laser as the light source of the IFOG since its frequency drift is usually within $1 \mathrm{ppm}[10,11]$. In contrast, the wavelength stability of the broadband light source is tens of ppm. As the scale factor stability directly depends on the performance of the light source, it can be improved by two orders of magnitude with laser instead of the SLD or SFS. However, the laser-driven IFOG reintroduces coherent backscattering, polarization coupling, and Kerr effect because of its narrow linewidth [12, 13]. Although the semiconductor lasers with the linewidth of tens of megahertz have been proved that they can drive the IFOG directly, it is still necessary to use broader laser for further improvement [14]. While multi-mode laser introduces more relative intensity noise (RIN) [15], some kinds of broadband lasers of gigahertz have worse wavelength stabilities. Alternatively, a few methods of broadening laser spectrum are put forward. Laser frequency modulation through dithering of the injection current can broaden the spectrum and reduce the IFOG noise to $0.033 \% / \mathrm{h}$, but it degrades the wavelength stability through coupling between the output and wavelength [16]. Its bias instability of $1.4^{\circ} \mathrm{h}$ is also frustrating. At present, external phase modulation is one of the best techniques $[10,11]$. Since the modulation process occurs after the laser is generated, it does not destroy the wavelength stability as direct modulation. In addition to the application in the IFOG, it has also been successfully applied to suppress stimulated Brillouin scattering (SBS) threshold [17]. By choosing Gaussian white noise $(\mathrm{GWN})$ as the driving signal, the spectrum does not contain sidebands or sub-peaks, and the effect of broadening and optical carrier suppression is outstanding, which is the most competitive choice compared with others such as the sinusoidal signal and pseudo-random bit sequence (PRBS) waveform [11].

According to the above background, this paper studies on the closed-loop IFOG driven by laser and improved by the external phase modulation. By studying the external phase modulation of the GWN, its effect of spectrum broadening is confirmed. An all-digital closed-loop FOG based on four-state modulation (FSM) is built to avoid temperature instability of the multifunction integrated-optic chip (MIOC) and drift caused by the electronic circuit in closed-loop demodulation. The test results show that the noise and drift of the IFOG driven by modulated laser reach $0.0038 \% / \mathrm{h}$ and $0.017 \% \mathrm{~h}$, which are $62 \%$ and $66 \%$ higher than those without modulation respectively, and the performance of drift has reached the level of the SLD. As there is no temperature control or vibration isolation, better results can also be available by overmodulation, signal processing, and temperature modeling.

\section{External phase modulation of the GWN}

Laser linewidth can be broadened by sending its output through an electro-optic phase modulator (EOM) and loading radio-frequency (RF) signal on the EOM to change the phase characteristics of the optical spectrum as shown in Fig. 1. The GWN is a preferred driving signal for spectrum broadening as explained above, especially in the IFOG.

The relationship between the phase noise and filed spectrum has been extensively studied [18]. The specific calculation methods among corresponding parameters are shown in Fig. 2. It includes the amplitude $E(t)$, the field spectrum $S(f)$, the phase difference $\Delta \phi_{\tau}(t)$, the frequency noise spectrum $S_{F}(f)$, the phase error variance $\sigma_{\phi}(\tau)^{2}$, and the differential phase spectrum 
$S_{\Delta \phi_{\tau}}(f)$. Equations (1) - (6) listed in Table 1 are used to relate those to each other and calculate
$S(f) . \mathscr{F}[*]$ represents the Fourier transform and $\langle *\rangle$ is the ensemble average.

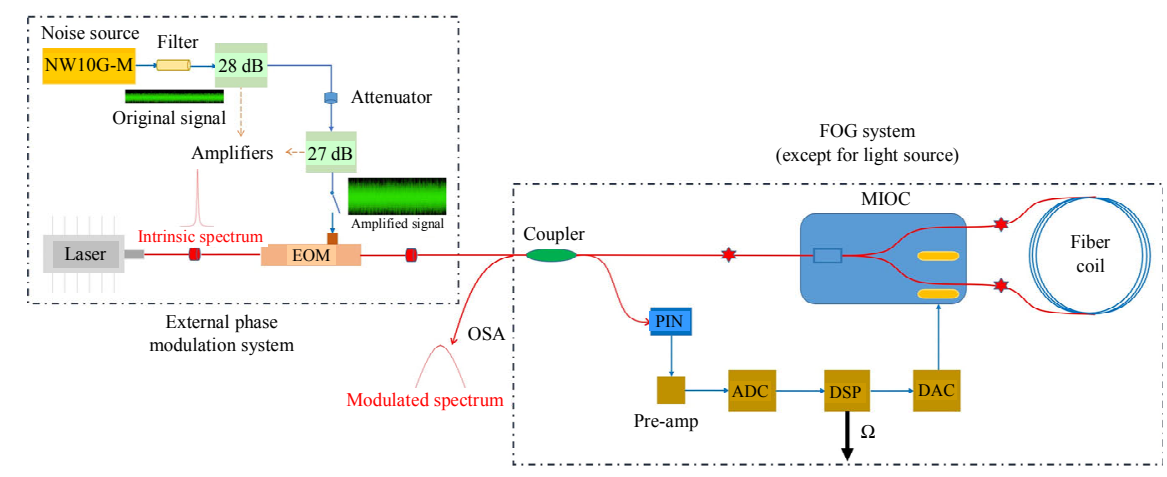

Fig. 1 Schematic diagram of linewidth broadening with the external phase modulation and IFOG.

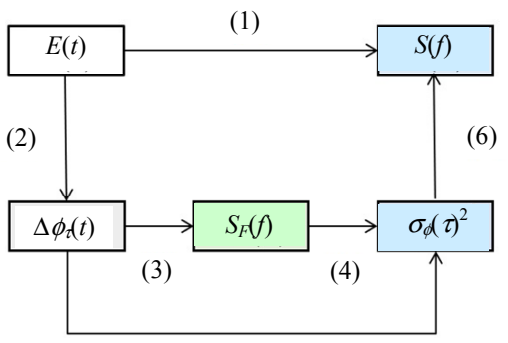

(5)

Fig. 2 Relations among the corresponding parameters.

Table 1 Equations relating the corresponding parameters to each other.

\begin{tabular}{c}
\hline Equation \\
\hline$S(f)=\left\langle\mid f[E(t)]^{2}\right\rangle$ \\
$\Delta \phi_{\tau}(t)=\phi_{n}(t)-\phi_{n}(t-\tau)$ \\
$S_{\Delta \phi_{\tau}}(f)=4\left(\frac{\sin (\pi f \tau)}{f}\right)^{2} S_{F}(f)$ \\
$\sigma_{\phi}(\tau)^{2}=\int_{0}^{\infty} S_{\Delta \phi_{\tau}}(f) \mathrm{d} f$ \\
$\sigma_{\phi}(\tau)^{2}=\left\langle\Delta \phi_{\tau}(t)^{2}\right\rangle$ \\
$S(f)=\mathscr{T}\left[\exp \left(-\frac{\sigma_{\phi}(\tau)^{2}}{2}\right)\right]$
\end{tabular}

As the EOM imposes phase noise on the field, the phase noise spectrum $S_{\varphi}(f)$ expressed as (7) should be substituted into (3):

$$
S_{\varphi}(f)=\frac{S_{F}(f)}{f^{2}}
$$

where $f$ is the Fourier frequency. Assuming that the bandwidth of the GWN is $f_{c}$ and the power spectral density (PSD) is $S_{V}$, the electrical disturbance is converted to phase disturbance in the field by the EOM, and its PSD is expressed as

$$
S_{E}=\pi^{2} S_{V} / V_{\pi}^{2}
$$

where $V_{\pi}$ is the half-wave voltage of the EOM. By combining the above equations, the autocorrelation function of $E(t)$ is expressed as

$$
R_{E}(\tau)=E_{0}^{2} \exp \left(\mathrm{i} 2 \pi v_{0} \tau\right) \exp \left\{-S_{E} f_{c}\left[1-\sin \mathrm{c}\left(2 f_{c} \tau\right)\right]\right\}
$$

where $E_{0}$ is the constant amplitude of the laser field, and $\tau$ is the random fluctuation time of the phase. The broadened spectrum with $v_{0}$ as the central frequency can be obtained by calculating the Fourier transform of (9). By increasing the PSD of the GWN under the same bandwidth, the spectrum asymptotically becomes a smooth Gaussian with the broader linewidth and less optical carrier as shown in Fig. 3(a). Improving the bandwidth as Fig. 3(b) also has the same effect. After further statistical analysis, the linewidth of the broadened component $\Delta v_{b}$ and the residual carrier of the spectrum $\Delta p_{c}$ are expressed as

$$
\begin{gathered}
\Delta v_{b}=\sqrt{\frac{8 \ln 2}{\pi} S_{E} f_{c}^{3}} \\
\Delta p_{c}=\mathrm{e}^{-S_{E} f_{c}} .
\end{gathered}
$$

Therefore, in order to achieve the excellent modulation effect, the GWN of the high power and modulation system of the wide bandwidth is necessary. In the experimental external phase modulation system in Fig. 1, the bandwidths of the 
RF driving signal, amplifier chain, and EOM are about $10 \mathrm{GHz}$, which is a choice under comprehensive consideration of the effect and cost. The EOM with $V_{\pi}$ of $3.7 \mathrm{~V}$ is driven by Noisewave NW10G-M, and a broadband GWN source also used in [11]. Its noise power is $-17 \mathrm{dBm}$, a lower value for spectrum broadening, which is supposed to be amplified before entering the EOM. The combination of a $10 \mathrm{GHz}$ low-pass filter and two-stage amplifiers, namely G3H84 and ZVA-183W+, is used to amplify the GWN power to $28 \mathrm{dBm}$, which is also the maximum RF input power of the EOM. An attenuator is used to protect the modulation system and adjust the input power of ZVA-183W+. By substituting the above parameters into (9), the optical spectra with and without modulation are calculated as shown in the Fig. 4(a). The intrinsic laser spectrum of the Lorentz shape is broadened to

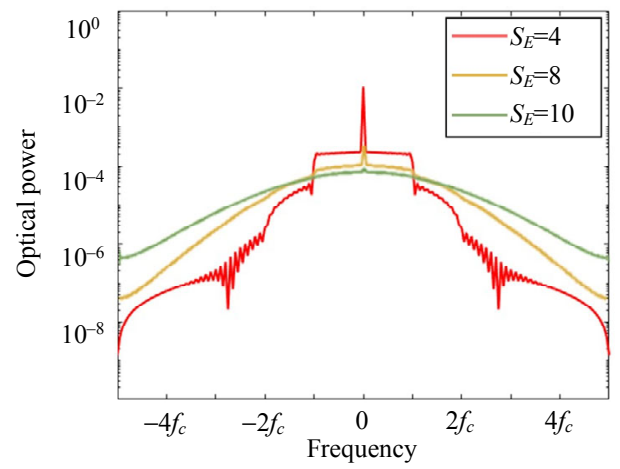

(a) the Gaussian spectrum of about $50 \mathrm{GHz}$. The actual test results measured by Yokogawa AQ6317C are shown in Fig.4(b), where the red line is the optical spectrum analyzer (OSA) impulse response of intrinsic laser with the true linewidth of $12 \mathrm{MHz}$ measured by the delayed self-heterodyne interferometer. The linewidth of its broadening component is more than $40 \mathrm{GHz}$ as fitted, which is close to the theoretical result, but there is residual carrier remained. The reason for this phenomenon is complex, which may be limited by the resolution of the OSA, the nonlinear modulation response of the EOM, voltage saturation of the GWN signal during amplification [11], etc. However, our theory still has an important guiding role, and the measurement results of the OSA and IFOG in the next section also prove that the spectrum broadening is effective.

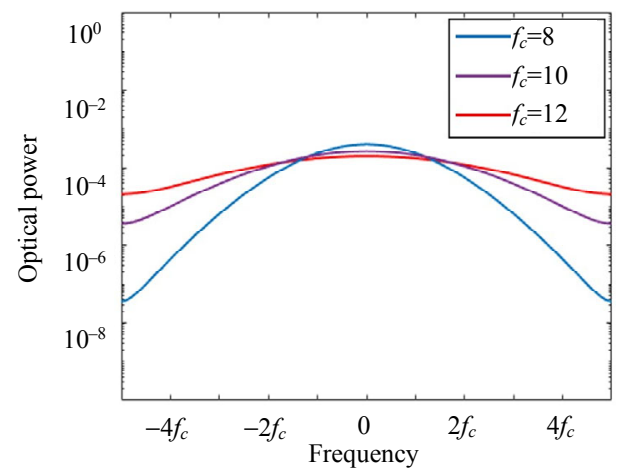

(b)

Fig. 3 Modulated optical spectra: (a) with increasing PSD and (b) with increasing bandwidth.

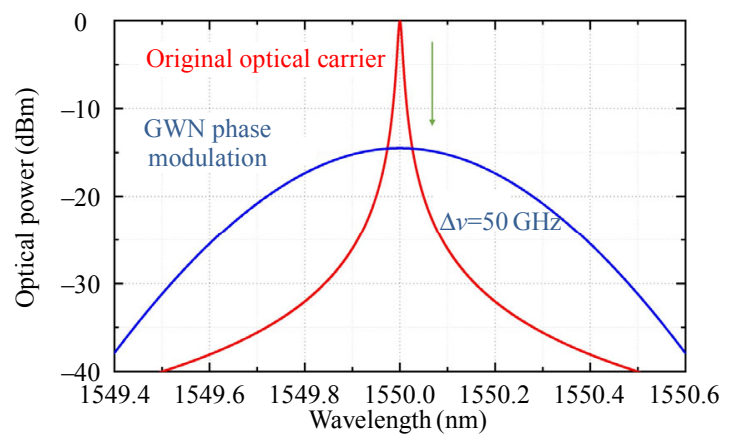

(a)

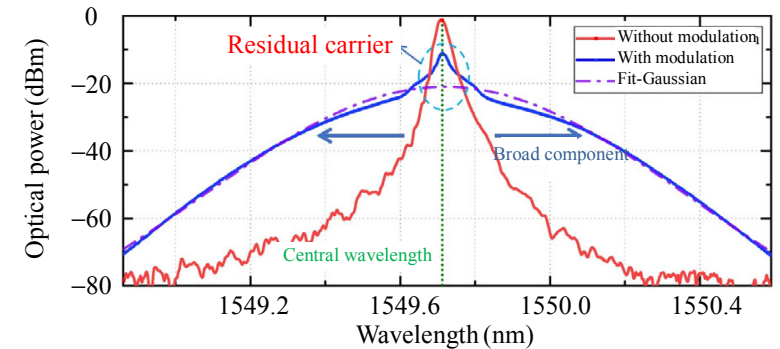

(b)

Fig. 4 Spectra with and without modulation: (a) simulation and (b) experiment.

At present, the EOM of $40 \mathrm{GHz}$ has been mature and mass produced, and the saturated output power of the RF signal can also be specially customized and increased, so the effect of laser spectrum 
broadening could reach a higher level. Therefore, the application of this technology will greatly improve the performance of the laser-driven IFOG.

\section{Experiment}

\subsection{IFOG system}

An all-digital closed-loop IFOG system using laser with the external phase modulation as the light source is established as Fig. 5. Except for the light source and modulation system introduced above, the IFOG is consisted of a coupler, a detector, a prime amplifier, an analog-to-digital converter (ADC), a digital-to-analog converter (DAC), and a digital signal processing (DSP) circuit, and the MIOC consists of a Y-waveguide, a polarizer and two modulators, and a fiber coil made of $1 \mathrm{~km}$ of polarization maintaining fiber and wound on a coil of $8 \mathrm{~cm}$ in diameter. The broadened laser is sent to the coupler and then to the MIOC where it is divided into two beams of light with opposite propagation directions in the fiber coil to form a Sagnac interferometer. After the lights interfere, the signal is detected and amplified by the pre-amplifier and then processed by the closed-loop control algorithm to generate the modulation signals which are used to drive the phase modulators in the MIOC. At the same time, the accurate rotational rate signals of the inertial space are output to the computer through the serial port.

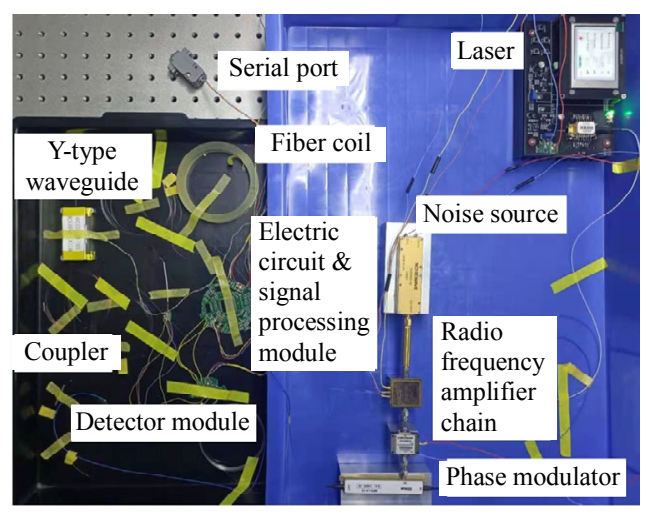

Fig. 5 Experimental system of the laser-driven IFOG.

To evaluate the performance of the laser-driven IFOG accurately and avoid errors in the cover-up, the system is tested on an indoor optical platform without any temperature control or extra vibration isolation, with the external phase modulator system in the blue area and the other devices of the IFOG in the black one.

\subsection{Closed-loop detection scheme}

For the closed-loop IFOG, its scale factor is expressed as

$$
K=\frac{2 \pi L D}{\lambda c} \cdot \frac{1}{K_{m} \cdot K_{\mathrm{DA}}}
$$

where $c$ is the speed of light in vacuum. In addition to the decisive factor, the average wavelength of the light source $\lambda$, the scale factor also depends on the length $L$ and diameter $D$ of the fiber coil, the modulation coefficient $K_{m}$ of the MIOC, and the gain $K_{\mathrm{DA}}$ of the DAC and amplifier. The stability of $\lambda$ has been improved by using laser. On the other hand, precise temperature modeling of the fiber coil can reduce the influence of $L$ and $D$ on the scale factor stability to a very low level, not to mention that there are materials with smaller thermal expansion coefficient to choose.

The closed-loop detection scheme not only improves the dynamic range of the IFOG, but also overcomes the temperature drift to a certain extent. However, its scale factor stability is also affected by the gain errors of closed-loop feedback mentioned above as $K_{m}$ and $K_{\mathrm{DA}}$, where the $V_{\pi}$ variation of phase modulators in the MIOC occupies the main reason which can reach $5 \%$ to $8 \%$ in the full temperature range of $-40{ }^{\circ} \mathrm{C}$ to $+60{ }^{\circ} \mathrm{C}$. It is necessary to control the closed-loop feedback gain so that the modulation phase corresponding to the step-wave reset voltage is always $2 \pi$. In the first feedback loop, $\varphi_{b}$ is generated to compensate the Sagnac phase $\varphi_{s}$ caused by rotation, and the FSM is adopted to realize the precise control of the feedback gain and compensate the gain error at any input rotation rate [19].

In the FSM, the phase modulator applies four different bias modulation states to the light for each 
modulation period and the form is expressed as

$$
\Delta \varphi(t)=\left\{\begin{array}{c}
\varphi_{0}, \quad 0 \leqslant t<\frac{\tau}{2} \\
a \varphi_{0}, \frac{\tau}{2} \leqslant t<\tau \\
-\varphi_{0}, \quad \tau \leq t<\frac{3 \tau}{2} \\
-a \varphi_{0}, \frac{3 \tau}{2} \leqslant t<2 \tau
\end{array}\right.
$$

where $\tau$ is the delay time of the fiber coil, $\varphi_{0}$ is the bias phase in the first and third states, $a$ is the gain of the bias phase in the second and fourth states and the requirement they should satisfy is $\cos \left(\varphi_{0}\right)=\cos \left(a \varphi_{0}\right)$. Generally, $\varphi_{0}=\pi / 2$ and $a=3$. When the IFOG is stationary, its output is usually a straight line including error pulses with a frequency of $1 / \tau$ as shown in Fig. 6. As the gain of the modulation channel becomes $(1+\varepsilon)$ times of the original, an error signal is generated in Fig. 7(a).When the IFOG rotates, its working point

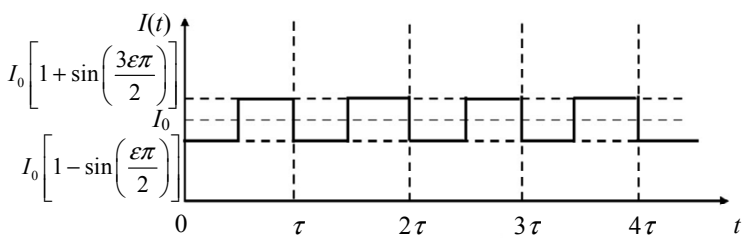

(a) moves and the output becomes a square wave with the same frequency as the modulated square wave in Fig. 7(b). The output signal in the actual working state is usually the superposition of them as shown in Figs. 7(c) and 6 with blue lines. The corresponding light intensities with $\varphi_{b}$ at the four modulation states are expressed as follows:

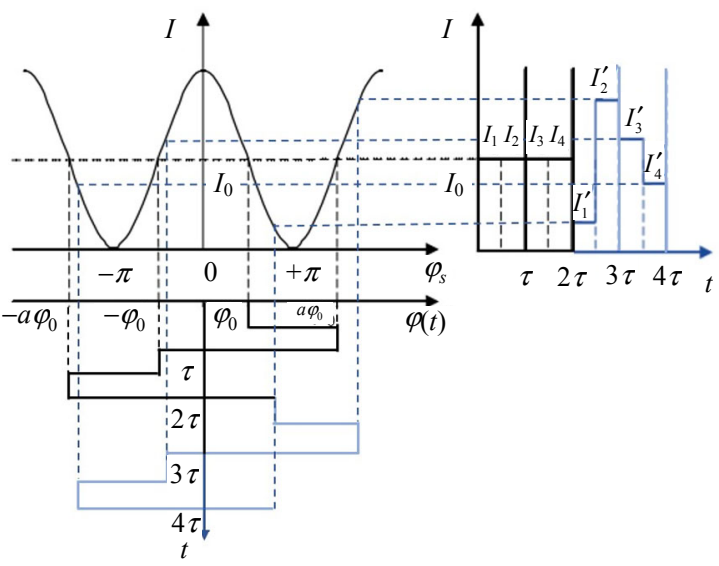

Fig. 6 Principle of the FSM in the IFOG.

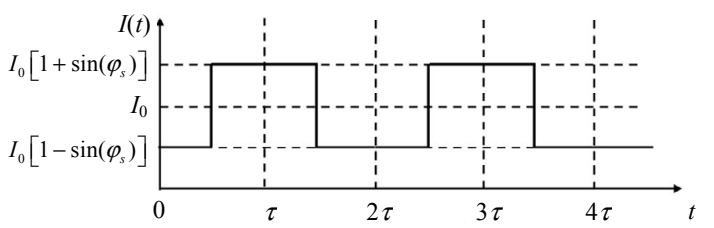

(b)

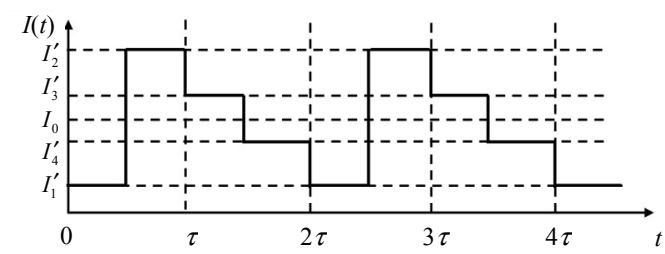

(c)

Fig. 7 Output signals of the IFOG with the FSM: (a) rotation rate signal input, (b) gain of closed-loop detection changes, and (c) actual working state with superposition.

$$
\begin{aligned}
I_{1}^{\prime} & =I_{0}\left\{1+\cos \left[\varphi_{s}+(1+\varepsilon)\left(\frac{\pi}{2}+\varphi_{b}\right)\right]\right\} \\
& \approx I_{0}\left[1-\varepsilon\left(\frac{\pi}{2}+\varphi_{b}\right)\right] \\
I_{2}^{\prime} & =I_{0}\left\{1+\cos \left[\varphi_{s}+(1+\varepsilon)\left(\frac{3 \pi}{2}+\varphi_{b}\right)\right]\right\} \\
& \approx I_{0}\left[1+\varepsilon\left(\frac{3 \pi}{2}+\varphi_{b}\right)\right]
\end{aligned}
$$

$$
\begin{aligned}
I_{3}^{\prime} & =I_{0}\left\{1+\cos \left[\varphi_{s}+(1+\varepsilon)\left(-\frac{\pi}{2}+\varphi_{b}\right)\right]\right\} \\
& \approx I_{0}\left[1+\varepsilon\left(-\frac{\pi}{2}+\varphi_{b}\right)\right] \\
I_{4}^{\prime} & =I_{0}\left\{1+\cos \left[\varphi_{s}+(1+\varepsilon)\left(-\frac{3 \pi}{2}+\varphi_{b}\right)\right]\right\} \\
& \approx I_{0}\left[1-\varepsilon\left(-\frac{3 \pi}{2}+\varphi_{b}\right)\right]
\end{aligned}
$$


where $I_{0}$ is the average detected signal intensity when the phase modulation is turned off. The output of the IFOG presents a ladder shape and the feedback gain error can be calculated as

$$
\Delta I=I_{2}^{\prime}-I_{1}^{\prime}+I_{4}^{\prime}-I_{3}^{\prime}=4 I_{0} \varepsilon \pi \text {. }
$$

Then the gain error is digitally integrated and the feedback algorithm generates the appropriate phase step and compensation. It is believed that the performance will be better than $1 \mathrm{ppm}$ by using laser as the light source with a frequency drift of tens of megahertz and introducing the FSM scheme which is tested in the next section.

\subsection{Experimental results}

The performance of the IFOG driven by broadened laser should be compared with the intrinsic laser and SLD simultaneously to study the improvement with modulation and the potential for laser replacing broadband light source. The IFOG system is placed in the room and measures the local component of the earth's rotation rate. The results in 100 minutes are shown in Fig. 8. After broadening the laser spectrum, the noise and drift of the IFOG have been significantly improved. The Allan variances in Fig. 8(d) show that the angular random walk noise is improved from $0.01 \% / \sqrt{h}$ to

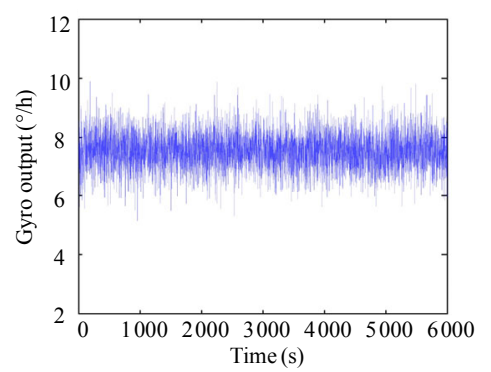

(a)

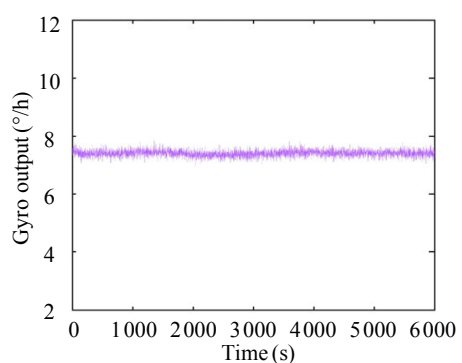

(c)
$0.0038 \% \sqrt{\mathrm{h}}$ and the drift is improved from $0.05 \% \mathrm{~h}$ to $0.017 \%$ h, which are $62 \%$ and $66 \%$ better, respectively. The noise is still relatively high compared with $0.0018 \% \sqrt{\mathrm{h}}$ of the SLD in the same IFOG system, but its performance could be promoted by using the less loss optical fiber, which has more influence on the laser-driven IFOG than the broadband light source because of coherent backscattering [12]. Furthermore, the results of the SLD are optimized by special digital signal processing beforehand, but the results of the laser-driven IFOG as shown in Fig. 8(b) are measured directly to avoid concealing the error, so its noise is expected to be reduced by several times hopefully with targeted treatment. On the other hand, the drift of the output in Fig. $8(\mathrm{c})$ is $0.014 \%$ h, which is almost the same as the test results in Fig. 8(b). These results mean that the polarization coupling has been suppressed to the level as other errors [13]. In this way, continuing to improve the spectral width will indeed improve its performance to a certain extent, but the effect diminishes significantly. It inspires the use of the light source with the relatively narrow spectral width instead of the SLD or SFS to achieve the lower cost and better scale factor stability for the medium and low-precision IFOG.

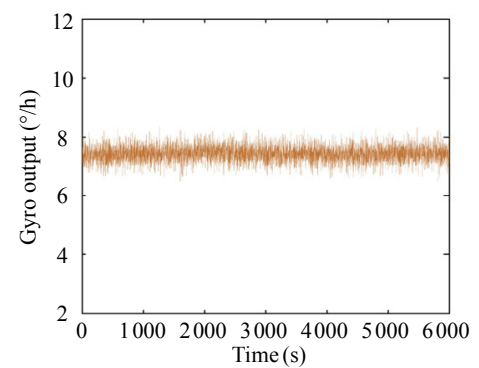

(b)

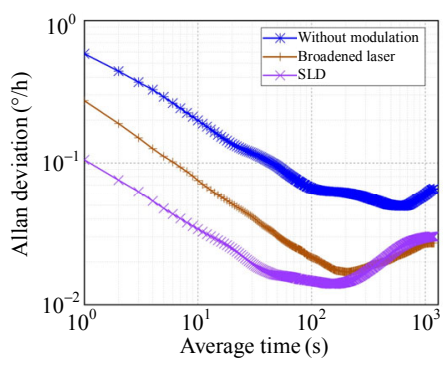

(d)

Fig. 8 Test results of the IFOG in 100 minutes with different light sources: (a) laser without modulation, (b) broadened laser, (c) SLD, and (d) measured Allan variances. 
In addition, the specific scale factor stability of the laser-driven IFOG is measured. It is installed on the rotating platform setting the rotation rate to $0 \% \mathrm{~s}$, $\pm 10 \% \mathrm{~s}, \pm 30 \% \mathrm{~s}, \pm 60 \% \mathrm{~s}, 90 \% \mathrm{~s}$, and $\pm 120 \% \mathrm{~s}$ respectively for 10 times in total. Digital outputs of the IFOG at different rotation rates are shown in Fig. 9. As the introduction of the external phase modulation and FSM will not change the scale factor greatly, digital outputs of the three conditions almost coincide in Fig. 9, while the tested stability is not the case. The scale factor is obtained by fitting the digital output of each group, and the stability is the standard deviation of the ten scale factors which is calculated as $1.88 \mathrm{ppm}$ when directly driven by laser. It should be noted that this value is relatively conservative due to the influence of noise and drift. Therefore, the scale factor stability is improved to $0.67 \mathrm{ppm}$ when the laser spectrum is modulated and broadened without degrading its frequency stability. After introducing the FSM technology, it is further improved to $0.38 \mathrm{ppm}$, an exciting result for the research of the IFOG. All tests meet the requirements of GJB 2426A-2004. Although the noise, volume, and power consumption of the IFOG driven by broadened laser still need improvement at present, its stability of the scale factor has an overwhelming advantage compared with the traditional IFOG and therefore enhances its competitiveness with the RLG.

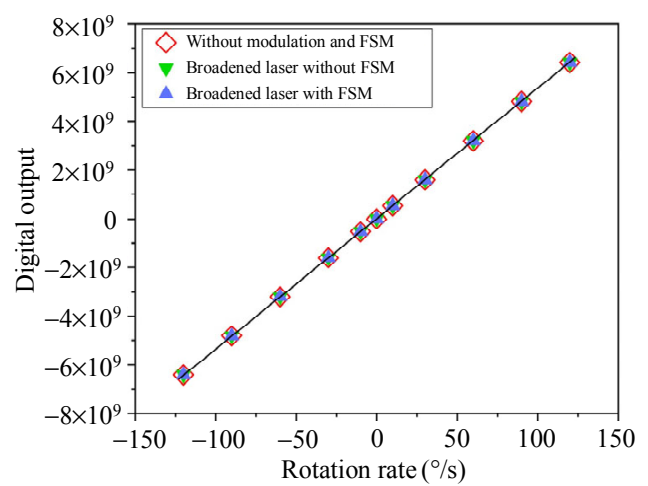

Fig. 9 Digital output of the laser-driven IFOG at different rotation rates.

\section{Conclusions}

The laser spectrum broadening method based on the external phase modulation of the GWN is studied. After shaping and amplifying the GWN signal generated from the noise source, the EOM is driven to broaden the spectrum and realize the broadening component of more than $40 \mathrm{GHz}$ with a small residual carrier. A closed-loop IFOG with the FSM is established. With the broadened laser, the angular random walk noise is $0.0038 \% \mathrm{Jh}$ and the drift is $0.017 \%$ h, which are $62 \%$ and $66 \%$ better than those without modulation, respectively. On the premise of ensuring that the stability of the scale factor is improved by two orders of magnitude than the traditional IFOG as $0.38 \mathrm{ppm}$, the drift is also the same as that driven by the SLD. Furthermore, the relatively high noise can be further improved and will not have a great impact on applications in the future. As the phase modulation technology is getting more and more mature which is hopeful to be integrated into the IFOG in the foreseeable future and the performance of the specially designed electronic equipment will also be improved, this paper shows that laser has a great potential as the the light source of the IFOG to enhance its competitiveness in the inertial device market.

\section{Acknowledgment}

The authors are grateful to all of the colleagues who participated in this research.

Open Access This article is distributed under the terms of the Creative Commons Attribution 4.0 International License (http://creativecommons.org/licenses/by/4.0/), which permits unrestricted use, distribution, and reproduction in any medium, provided you give appropriate credit to the original author(s) and the source, provide a link to the Creative Commons license, and indicate if changes were made.

\section{References}

[1] H. J. Arditty and H. C. Lefevre, "Theoretical basis of Sagnac effect in fiber gyroscopes," in the First International Conference MIT, USA, November 9-11, 1981, pp. 44-51.

[2] Z. Zhou, Z. W. Tan, X. Y. Wang, and Z. Y. Wang, "Experimental analysis of the dynamic north-finding 
method based on a fiber optic gyroscope," Applied Optics, 2017, 56(23): 6504-6510.

[3] W. B. Gan, W. B. Hu, F. Liu, J. G. Tang, S. Li, and Y. Yang, "Bridge continuous deformation measurement technology based on fiber optic gyro," Photonic Sensors, 2016, 6(1): 71-77.

[4] Q. Wang, J. Xie, C. Yang, C. He, X. Wang, and Z. Wang, "Step angles to reduce the north-finding error caused by rate random walk with fiber optic gyroscope," Applied Optics, 2015, 54(30): 8944-8950.

[5] Z. Y. Zhang and C. T. Liu, "Fiber optic gyroscope dynamic north-finder algorithm modeling and analysis based on Simulink," Photonic Sensors, 2017, 7(3): 283-288.

[6] H. C. Lefevre, "The fiber-optic gyroscope: challenges to become the ultimate rotation-sensing technology," Optical Fiber Technology, 2013, 19(6): 828-832.

[7] I. R. Edu, R. Obreja, and T. L. Grigorie, "Current technologies and trends in the development of gyros used in navigation applications - a review," in Proceedings of the 5th WSEAS International Conference on Communications and Information Technology, Greece, 2011, pp. 63-68.

[8] L. Zhang, S. Ye, F. Liu, and S. D. Zhou, "Detection method for the singular angular velocity intervals of the interferometric fiber optic gyroscope scale factor," Optik, 2016, 127(22): 10412-10420.

[9] S. W. Lloyd, M. J. F. Digonnet, and S. H. Fan, "Near shot-noise limited performance of an open loop laser-driven interferometric fiber optic gyroscope," SPIE, 2011, 7753: 7753A3.

[10] J. Chamoun and M. J. F. Digonnet, "Pseudorandom-bit-sequence phase modulation for reduced errors in a fiber optic gyroscope," Optics Letters, 2016, 41(24): 5664-5667.

[11] J. Chamoun and M. J. F. Digonnet, "Aircraft- navigation-grade laser-driven FOG with Gaussian-noise phase modulation," Optics Letters, 2017, 42(8): 1600-1603.

[12] S. W. Lloyd, M. J. F. Digonnet, and S. H. Fan, "Modeling coherent backscattering errors in fiber optic gyroscopes for sources of arbitrary line width," Journal of Lightwave Technology, 2013, 31(13): 2070-2078.

[13] J. N. Chamoun and M. J. F. Digonnet, "Noise and bias error due to polarization coupling in a fiber optic gyroscope," Journal of Lightwave Technology, 2015, 33(13): 2839-2847.

[14] J. N. Chamoun, A. Evans, F. A. Mosca, and M. J. F. Digonnet, "Low noise and low drift in a laser-driven fiber optic gyroscope with a 1-km coil," SPIE, 2014, 9157: 91570E.

[15] I. S. Kim, P. Tantaswadi, and J. Blake, "Coherencecollapsed $1.3-\mu \mathrm{m}$ multimode laser diode for the fiber-optic gyroscope," Optics Letters, 1995, 20(7): 731-733.

[16] T. Komljenovic, M. A. Tran, M. Belt, S. Gundavarapu, D. J. Blumenthal, and J. E. Bowers, "Frequency modulated lasers for interferometric optical gyroscopes," Optics Letters, 2016, 41(8): 1773-1776.

[17] B. Anderson, A. Flores, R. Holten, and I. Dajani, "Comparison of phase modulation schemes for coherently combined fiber amplifiers," Optics Express, 2015, 23(21): 27046-27060.

[18] K. Kikuchi, "Characterization of semiconductorlaser phase noise and estimation of bit-error rate performance with low-speed offline digital coherent receivers," Optics Express, 2012, 20(5): 5291-5302.

[19] D. He, Y. J. Wu, Y. L. Li, Z. R. Zhang, C. Peng, and Z. B. Li, "Stability improvement enabled by four-state modulation in dual-polarization fiber optic gyroscopes," Optics Communications, 2019, 452: 68-73. 